\title{
Blickpunkt: Rücküberweisungen : Umfrage unter serbischstämmigen Migranten in der Schweiz
}

Mathias Lerch

\section{(2) OpenEdition}

12 Journals

\section{Electronic version}

URL: http://journals.openedition.org/sjep/336

DOI: 10.4000/sjep.336

ISSN: 1663-9677

\section{Publisher}

Institut de hautes études internationales et du développement

\section{Printed version}

Date of publication: 1 décembre 2008

Number of pages: 169-174

ISBN: 978-2-940415-08-3

ISSN: 1660-5926

\section{Electronic reference}

Mathias Lerch, «Blickpunkt: Rücküberweisungen : Umfrage unter serbischstämmigen Migranten in der Schweiz », Schweizerisches Jahrbuch für Entwicklungspolitik [Online], 27-2 | 2008, Online erschienen am: 31 Mai 2010, abgerufen am 07 September 2020. URL : http://journals.openedition.org/sjep/336 DOI : https://doi.org/10.4000/sjep.336 


\title{
Blickpunkt
}

\section{Rücküberweisungen: Umfrage unter serbischstämmigen Migranten in der Schweiz}

\author{
Mathias Lerch*
}

D ass die Migration ein Entwicklungspotenzial für die Herkunftsländer der Migrantinnen und Migranten birgt und einen Beitrag zur Armutsverringerung leisten kann, ist heute unbestritten'. Vor dem Hintergrund politischer oder wirtschaftlicher Krisen, wie sie für den seit 1992 in Serbien andauernden Umbruch bezeichnend sind, kann der Umfang der von Migrantinnen und Migranten in die Heimat überwiesenen Gelder - der sogenannten Remittances sogar noch zunehmen². Diese in der Zahlungsbilanz als „private Übertragungen" definierten Rücküberweisungen ${ }^{3}$ stellen einen bedeutenden Anteil an den internationalen Finanzflüssen dar, welcher den Umfang der öffentlichen Entwicklungshilfe übersteigt. Damit kommt der Kenntnis des ökonomischen Verhaltens von Migrantinnen und Migranten gegenüber ihrem Herkunftsland und der Faktoren, die für die Praxis der Rücküberweisungen entscheidend sind, eine unübersehbare Bedeutung zu. Dies gilt insbesondere in Bezug auf die Harmonisierung und die Kohärenz der Migrations- und der Entwicklungshilfepolitiken.
Bevölkerungsumfragen unter Migrantinnen und Migranten sind ein flexibles Messinstrument: Einerseits erlauben sie es, die bis heute lückenhaften makroökonomischen Schätzungen in Bezug auf die Rücküberweisungsflüsse zu verfeinern und den Umfang der verschiedenen bilateralen Flüsse aus einem Einwanderungsland - die sogenannten Remittance-Korridore - zu ermitteln. Andererseits liefern Umfragen qualitative Informationen über die Praktiken der Migrantinnen und Migranten bei der Überweisung von Geldern sowie über den Grad ihrer Integration im Gastland". Diese Angaben dürften bei der Ausrichtung der Entwicklungshilfepolitik hilfreich sein und die Komplementarität zwischen privater und öffentlicher Hilfe fördern.

Der vorliegende Beitrag erörtert die wichtigsten Rückschlüsse aus der ersten Umfrage über die Rücküberweisungspraktiken von in der Schweiz wohnenden Migrantinnen und Migranten. Diese Umfrage wurde gezielt unter Personen serbischer Herkunft durchgeführt, das heisst unter serbischsprachigen Personen. Es handelt sich dabei also um eine

* Wissenschaftlicher Mitarbeiter beim Laboratoire de démographie et d'études familiales, Faculté des sciences économiques et sociales, Universität Genf.

1 R.H. Adams and J. Page, „Do international Migration and Remittances Reduce Poverty in Developing Countries?”, World Development, Bd. 33, Nr. 10, 2005, S. 1645-1669.

2 M. Schrooten, Bringing Home the Money: What Determines Workers' Remittances to Transition Countries, Discussion Paper Series A, Nr. 466, Institute of Economic Research (IER), Tokyo, Hitotsubashi University, 2005.

3 Hierbei handelt es sich um die Gesamtheit der Geld- oder Gütertransfers von Haushalten im Inland hin zu Haushalten im Herkunftsland innerhalb der letzten zwölf Monate vor der Durchführung der Umfrage, über die hier berichtet wird. Vgl. hierzu A. Alfieri and I. Havinga, Issue Paper: Definition of Remittances, Meeting of the United Nations Technical Subgroup on Movement of Persons - Mode 4, New York, United Nations, 22-24 February 2006.

4 M. Lerch, P. Wanner and W. Haug, The Measurement of Remittances in Industrialized Countries - or How to Limit Multiple Biases, Background Paper for the Joint UNECE/World Bank/US Census Bureau Expert Group Meeting on the Contribution of Household Surveys to Measuring Remittances, Suitland (United States), 14-15 January 2008. 
Untergruppe der Einwanderer serbisch-montenegrinischer Nationalität, wie sie in der Statistik der Schweiz aus dem Jahr 2006 erscheinen. Die zahlenmässig bedeutende Gruppe der albanischsprachigen (hauptsächlich kosovarischen) oder montenegrinischen Einwanderer wird durch die Umfrage nicht abgedeckt ${ }^{5}$. Eine präzise Identifizierung der untersuchten Population anhand der offiziellen Statistiken ist folglich nicht möglich. Allerdings verdeutlichen die Statistiken den Grad der Abhängigkeit des Herkunftslandes als Ganzes von den Remittances: Diese beliefen sich für Serbien im Jahr 2005 auf 4129 Millionen Dollar. Damit ist Serbien der wichtigste Empfängerstaat von Rücküberweisungen unter den Balkanländern und rangiert weltweit unter den zehn wichtigsten Empfängern von Remittances (in Prozent des BIP)6.

\section{Besonderheiten der serbischen Migration und Umfrage unter Migrantinnen und Migranten in der Schweiz}

Die ersten serbischen Migranten kamen Anfang des 20. Jahrhunderts in die Schweiz. Anlass dafür war der Abschluss einer konsularischen Vereinbarung mit dem Königreich Serbien, die die Einwanderung im Rahmen der wirtschaftlichen, politischen und kulturellen Beziehungen zwischen den beiden Ländern erleichterte ${ }^{7}$. Ab 1967 wurde aufgrund des demografischen und ökonomischen
Drucks die zeitlich befristete Auswanderung von der 1945 eingesetzten jugoslawischen Regierung aktiv gefördert ${ }^{8}$. Mit der Einführung des Drei-Kreise-Modells durch die Schweiz im Jahre 1990, welches das ehemalige Jugoslawien aus der Gruppe der Rekrutierungsländer für Arbeitskräfte ausschloss, wurde indessen die wirtschaftliche Einwanderung aus Serbien erschwert ${ }^{9}$.

Infolge des Bürgerkrieges und des wirtschaftlichen Umbruchs hielt der starke Auswanderungsdruck jedoch an. In den 1980erund 1990er-Jahren verzeichnete die Gruppe der in der Schweiz wohnenden serbischen Staatsangehörigen ein starkes Wachstum. Sie zählte Ende 2007198365 Personen (einschliesslich Kosovarinnen und Kosovaren) ${ }^{10}$ und stellte mit 12 Prozent die grösste Gruppe unter der ausländischen Wohnbevölkerung, verglichen mit lediglich 2,3 Prozent im Jahr 1970. Der Grund für diesen Zuwachs liegt im Wandel der Migrationsgründe während der 1990er-Jahre: Während dieser Zeit wurden mehr Einreisen aus humanitären Gründen beziehungsweise im Rahmen des Familiennachzugs verzeichnet als früher ${ }^{11}$.

Verglichen mit anderen Bevölkerungsgruppen aus dem ehemaligen Jugoslawien (z. B. aus Kosovo und Mazedonien) zeichnet sich die serbischstämmige Bevölkerung in der Schweiz aus durch ihre Stabilität, durch die hohe Zahl an Familien sowie durch ein ver-

5 Zum Zeitpunkt der Umfrage bestand die Bevölkerungsgruppe serbisch-montenegrinischer Nationalität aus Personen serbischer, montenegrinischer oder kosovarischer Herkunft sowie aus Angehörigen weiterer ethnischer Minderheiten. In der Zwischenzeit sind die Herkunftsgebiete dieser drei Gruppen zu unabhängigen Staaten geworden. Zur Bezeichnung der ethnischen Gruppe, unter der die Umfrage durchgeführt wurde, wird im Folgenden das Attribut "serbischer Herkunft" bzw. „serbischstämmig" verwendet.

6 M. Schrooten, op. cit.

7 D. Mikic, "Historische Hintergründe zur Migration in die Schweiz", in Einblicke: Serbinnen und Serben in der Migration, Fachstelle für interkulturelle Fragen der Stadt Zürich, November 2001.

8 I.G. Molnar, "The Sociology of Migration from the Former Yugoslavia», New Community, Bd. 23, Nr. 1, 1997, S. 109-122.

9 D. Efionayi, J.M. Niederberger and P. Wanner, "Switzerland Faces Common European Challenges", Migration Information Source (MPI), Washington, DC, Migration Policy Institute, 2005, <http://www.migrationinformation. org/Feature/print.cfm?ID=284> (konsultiert am 10. Januar 2008).

10 Quelle: Bundesamt für Migration (BFM), <http://www.bfm.admin.ch/bfm/de/home/themen.html> >Statistiken $>$ Ausländerstatistik >Aktuelle Ergebnisse (konsultiert am 2. Juni 2008).

11 D.M. Gross, Immigration to Switzerland: The Case of the Former Republic of Yugoslavia, World Bank Policy Research Working Paper, Nr. 3880, Washington, DC, The World Bank, 2006. 
gleichsweise höheres Ausbildungsniveau und eine stärkere wirtschaftliche Integration $^{12}$. Damit dürfte das wirtschaftliche Gewicht der Migrantinnen und Migranten im heutigen Serbien am grössten sein.

Angesichts dessen hat das Staatssekretariat für Wirtschaft (SECO) ein Forschungsprojekt über die Remittance-Korridore zwischen der Schweiz und dem heutigen Serbien initiiert mit dem Ziel, die Transferpraktiken, die Effizienz des Bankensystems und die Verwendung der Gelder im Herkunftsland zu untersuchen. Die Informationen wurden im März 2006 im Rahmen einer telefonischen Umfrage bei 600 serbischstämmigen Haushalten in der Schweiz erhoben. Als Grundlage für die Stichprobennahme diente das Zentrale Ausländerregister, welches ein vollständiges Verzeichnis der Namen und Adressen der aus Serbien-Montenegro stammenden Personen in der Schweiz enthält. Allerdings können die Umfrageergebnisse nicht ohne Weiteres für die gesamte Gemeinschaft verallgemeinert werden, denn die präzise Definition der Stichprobenbasis das heisst der Referenzpopulation der Stichprobe - gestaltete sich mangels Angaben über die ethnische Zugehörigkeit der einzelnen Personen schwierig ${ }^{13}$.

\section{Umfang und Determinanten der Rücküberweisungen von serbischen Einwanderern in der Schweiz}

Die Analyse der Umfrageergebnisse hat gezeigt, dass die grosse Mehrheit der in der Schweiz wohnenden serbischstämmigen Personen wirtschaftliche Beziehungen mit ihrem Herkunftsland pflegen: Drei Viertel der befragten Haushalte haben Geld oder Güter in die Heimat geschickt. Der Median der überwiesenen Beträge liegt bei 3000 Franken pro Jahr und Haushalt in der Schweiz. In der Regel sind mehrere Empfänger begünstigt, hauptsächlich Grosseltern (45\%), Geschwister $(20 \%)$ oder im Herkunftsland zurückgebliebene Mitglieder der eigenen Familie (11\%).

Für die begünstigten Haushalte stellen diese Geldtransfers eine bedeutende Einnahmequelle dar: Der Betrag pro Empfänger in Serbien liegt im Median bei 1000 Franken jährlich; dies sind 20 Prozent mehr als der Schwellenwert für extreme Armut und entspricht der Hälfte der absoluten Armutsschwelle ${ }^{14}$. Tatsächlich verfolgen vier von fünf Haushalten, die Rücküberweisungen tätigen, mit ihren Geldtransfers das Ziel, einen Beitrag an den Erwerb von Konsumgütern des täglichen Bedarfs oder an Gesundheitskosten zu leisten.

Die bedeutende Neigung der serbischstämmigen Bevölkerung in der Schweiz, Geld in die Heimat zu überweisen, ist durch verschiedene Faktoren begründet. Zum einen besteht diese Neigung unabhängig von der Dauer des Aufenthalts in der Schweiz, auch wenn die Höhe der überwiesenen Beträge abnimmt, je länger die betreffenden Personen in der Schweiz leben. Dies ist ein bedeutender Unterschied zu der Mehrheit der anderen Einwanderergruppen, bei denen die Neigung, Rücküberweisungen in ihr Herkunftsland zu tätigen, mit zunehmender Länge des Aufenthalts in der Schweiz abnimmt. Ausserdem dürfte die in den 1990er-Jahren aufgrund der bewaffneten Auseinandersetzungen und des wirtschaftlichen Umbruchs verzeichnete Verschlechterung des Lebensstandards in Serbien die Bereitschaft der serbischstämmigen Einwanderer, ihr Herkunftsland wirtschaftlich zu unterstützen, (erneut) mobilisiert haben. Zum

12 M. Lerch, J. Dahinden and P. Wanner, Mapping Three Balkan Diasporas in Switzerland, Neuchâtel, Swiss Forum for Migration and Population Studies, 2005 (unveröffentlicht).

13 Vgl. M. Lerch, J. Dahinden and P. Wanner, Remittance Behaviour of Serbian Migrants in Switzerland, SFM Studies, Nr. 51, Neuchâtel, Swiss Forum for Migration and Population Studies, 2007.

14 World Bank, Serbia and Montenegro: Poverty Assessment, Report, Nr. 26011-YU, Bd. 2, Poverty Reduction and Economic Management Unit, Europe and Central Asia Region, Washington, DC, The World Bank, 2003. 
andern wird die Pflege der länderübergreifenden sozialen und wirtschaftlichen Beziehungen durch die geografische Nähe des Herkunfts- und des Gastlandes erleichtert. Diese Beziehungen sind für die Rücküberweisungen der serbischstämmigen Bevölkerung in der Schweiz von entscheidender Bedeutung: Wer nach wie vor über intakte Beziehungsnetze in Serbien verfügt, öfter nach Serbien reist und dort ein Bankkonto besitzt, ist tendenziell eher geneigt, Geld nach Serbien zu schicken, und überweist in der Regel auch höhere Beträge.

Indessen sind die serbischstämmigen Einwanderer in der Schweiz aufgrund ihrer Lebensumstände nur begrenzt in der Lage, ihr Herkunftsland finanziell zu unterstützen. Insbesondere die momentane Familienlebensphase und der Grad der wirtschaftlichen Integration sind für die Rücküberweisungspraktiken entscheidend. Im Gegensatz zu anderen Migrationskontexten weltweit sind finanziell gut gestellte serbischstämmige Haushalte in der Schweiz allerdings eher geneigt, Geld in ihr Herkunftsland zu schicken. Aber wie in den meisten anderen Einwanderungsländern lässt sich auch bei innen feststellen, dass die überwiesenen Beträge umso höher sind, je besser das Ausbildungsniveau und die wirtschaftliche Lage des jeweiligen Haushaltes sind. Auch unlängst eingebürgerte serbischstämmige Personen schicken mehr Geld in ihr Herkunftsland, wenn auch weniger häufig. Diese soziodemografischen Merkmale sind entscheidend für eine erfolgreiche Integration in den Arbeitsmarkt. Allerdings sind gerade bei jungen Einwandererfamilien den finanziellen Möglichkeiten zur Unterstützung des Herkunftslandes wegen der hohen Lebenskosten Grenzen gesetzt. Es überrascht deshalb nicht, dass Haushalte ohne beziehungsweise mit erwachsenen Kindern eine höhere Wahrscheinlichkeit aufweisen, Geld in die Heimat zu überweisen.
Die Nutzung der Bankkanäle schliesslich ist für die wirtschaftliche Entwicklung des Herkunftslandes von besonderer Bedeutung. Remittances, die über diese Kanäle ins Land fliessen, fördern die Finanzintermediation durch die Banken und mithin das Angebot an Investitionskrediten. Allerdings nutzt weniger als ein Fünftel der serbischstämmigen Haushalte bei ihren Rücküberweisungen formelle Kanäle, namentlich jene der Banken. Die Mehrheit der Geldsendungen erfolgt durch eigenhändige Übergabe. Dies ist nicht nur schneller, sondern gilt auch als sicherer als Banküberweisungen. Es ist durchaus möglich, dass das Einfrieren der Guthaben von Emigranten während der Milosevic-Ära und der Zusammenbruch der pyramidalen Bankensysteme ${ }^{15}$ in den 1990er-Jahren noch heute das Vertrauen in das lokale Bankenwesen belasten. Allerdings hat sich der Bankenmarkt in der Zwischenzeit stabilisiert, und seit dem Ende des Kosovo-Krieges haben zahlreiche ausländische Banken in Serbien Fuss gefasst.

Dass formelle Kanäle nur selten genutzt werden, ist zum Teil auf mangelnde Informationen zurückzuführen: Ältere Personen oder auch solche, die die Sprache des Gastlandes nur schlecht beherrschen, nutzen diese Kanäle am seltensten. Demgegenüber tätigen Serbinnen und Serben, deren Beziehungsnetz stärker in der Schweiz verankert ist, häufiger Banküberweisungen. Offensichtlich erhöhen sich die Kenntnisse über das heutige Bankenwesen mit zunehmender Integration. Die Kosten für eine Banküberweisung sind zwar niedriger als jene der auf Geldtransfers spezialisierten Agenturen, aber im Bankenwesen mangelt es an Transparenz: Häufig wissen die Schweizer Banken über die von den Empfängerbanken im Herkunftsland erhobenen zusätzlichen Spesen nicht Bescheid.

15 Bank Austria, Banking in Serbia and Montenegro, Extra Report, Economics Department, Bank Austria Creditanstalt AG, 2005. World Bank, op. cit. 
rer bestimmt. Und drittens bewirkt der Familiennachzug, dass weniger häufig RücküberIn Serbien, dem einst reichsten Land der Balkanregion, hat sich der Lebensstandard in den 1990er-Jahren aufgrund der Auseinandersetzungen und des wirtschaftlichen Umbruchs massiv verschlechtert. Dadurch wurde die Bereitschaft der serbischen Emigrantinnen und Emigranten, ihre Heimat zu unterstützen, erneut und in bedeutendem Umfang mobilisiert. Die Gelder, die serbischstämmige Einwanderer in der Schweiz in ihr Herkunftsland überweisen, lindern insbesondere unter der älteren Bevölkerung Serbiens die Armut, bewirken aber nur geringfügige Investitionen durch die Empfängerhaushalte.

Die Umfrageergebnisse haben ferner gezeigt, dass die Politiken der Gastländer den Umfang und die Wirkung der Rücküberweisung beeinflussen können. Erstens können sie die Bedeutung der länderübergreifenden Beziehungen der Migrantinnen und Migranten, wozu auch die Remittances gehören, unterstreichen ${ }^{16}$. Die Schaffung günstiger Voraussetzungen für die Aufrechterhaltung der Beziehungen zum Herkunftsland - etwa durch die Personenfreizügigkeit für Einwanderer oder durch die Möglichkeit für serbische Auswanderer, ihr Wahlrecht im Ausland auszuüben - könnte somit zum Wachstum dieser Finanzflüsse beitragen. Zweitens wird das Budget für die Unterstützung des Herkunftslandes massgeblich durch die Integrationsmöglichkeiten für Einwande-

weisungen getätigt und geringere Beträge überwiesen werden, denn die verfügbaren Mittel der Haushalte werden in erster Linie in die Ausbildung der Kinder investiert. Politische Massnahmen, die die individuelle Arbeitsmigration begünstigen, könnten zweifellos eine Zunahme der Remittance-Ströme bewirken. In Betracht zu ziehen wären ferner politische Interventionen, die auf die Preise für Geldtransfers abzielen. Die Information über formelle Geldüberweisungskanäle und ihre Vorteile ${ }^{17}$ könnte dazu beitragen, dass diese vermehrt genutzt werden. Dies würde bewirken, dass die Banken im Herkunftsland vermehrt als Finanzintermediäre auftreten könnten, wodurch sich das Angebot an Investitionskrediten vergrössern würde.

Bevölkerungsumfragen liefern indessen nicht nur qualitative Informationen. Sie können auch dazu beitragen, die nach wie vor lückenhaften Schätzungen über die bilateralen Remittance-Flüsse aus der Schweiz zu verfeinern. Die Untersuchung des wirtschaftlichen Verhaltens der Migrantinnen und Migranten gegenüber ihrem Herkunftsland anhand von Umfragen dürfte die Kohärenz zwischen Migrationspolitik und Entwicklungshilfepolitik fördern, denn die Merkmale der Migranten(haushalte) haben einen stärkeren Einfluss auf die Remittances und folglich auf die Auswirkungen der Migration im Herkunftsland als makroökonomische Faktoren ${ }^{18}$.

16 L.E. Guarnizo, "The Economics of Transnational Living“, International Migration Review, Bd. 37, Nr. 3, 2003, S. 666-699.

17 Eine Vorreiterrolle auf diesem Gebiet spielt beispielsweise die britische Regierung.

18 S.S. Russel, „Remittances from International Migration: A Review in Perspective“, World Development, Bd. 14, Nr. 6, 1986, S. 677-696. 


\section{Bibliographie}

Adams, R.H. and J. Page, „Do international Migration and Remittances Reduce Poverty in Developing Countries?", World Development, Bd. 33, Nr. 10, 2005, S. 1645-1669.

Alfieri, A. and I. Havinga, Issue Paper: Definition of Remittances, Meeting of the United Nations Technical Subgroup on Movement of Persons - Mode 4, New York, United Nations, 22-24 February 2006.

Bank Austria, Banking in Serbia and Montenegro, Extra Report, Economics Department, Bank Austria Creditanstalt $A G, 2005$.

Efionayi, D., J.M. Niederberger and P. Wanner, „Switzerland Faces Common European Challenges“, Migration Information Source (MPI), Washington, DC, Migration Policy Institute, 2005, <http://www.migrationinformation.org/ Feature/print.cfm?ID=284> (konsultiert am 10. Januar 2008).

Gross, D.M., Immigration to Switzerland: The Case of the Former Republic of Yugoslavia, World Bank Policy Research Working Paper, Nr. 3880, Washington, DC, The World Bank, 2006.

Guarnizo, L.E., „The Economics of Transnational Living", International Migration Review, Bd. 37, Nr. 3, 2003, S. 666-699.

Lerch, M., J. Dahinden and P. Wanner, Mapping Three Balkan Diasporas in Switzerland, Neuchâtel, Swiss Forum for Migration and Population Studies, 2005 (unveröffentlicht).

Lerch, M., J. Dahinden and P. Wanner, Remittance Behaviour of Serbian Migrants in Switzerland, SFM Studies, Nr. 51, Neuchâtel, Swiss Forum for Migration and Population Studies, 2007.

Lerch, M., P. Wanner and W. Haug, The Measurement of Remittances in Industrialized Countries - or How to Limit Multiple Biases, Background Paper for the Joint UNECE/World Bank/US Census Bureau Expert Group Meeting on the Contribution of Household Surveys to Measuring Remittances, Suitland (United States), 14-15 January 2008.

Mikic, D., „Historische Hintergründe zur Migration in die Schweiz", in Einblicke: Serbinnen und Serben in der Migration, Fachstelle für interkulturelle Fragen der Stadt Zürich, November 2001.

Molnar, I.G., , "The Sociology of Migration from the Former Yugoslavia“, New Community, Bd. 23, Nr. 1, 1997, S. 109-122.

Russel, S.S., „Remittances from International Migration: A Review in Perspective“, World Development, Bd. 14, Nr. 6, 1986, S. 677-696.

Schrooten, M., Bringing Home the Money: What Determines Workers' Remittances to Transition Countries, Discussion Paper Series A, Nr. 466, Institute of Economic Research (IER), Tokyo, Hitotsubashi University, 2005.

World Bank, Serbia and Montenegro: Poverty Assessment, Report, Nr. 26011-YU, Bd. 2, Poverty Reduction and Economic Management Unit, Europe and Central Asia Region, Washington, DC, The World Bank, 2003. 